\title{
Association of HO-1 and BRCA1 Is Critical for the Maintenance of Cellular Homeostasis in Prostate Cancer
}

\author{
Estefanía Labanca1,2, Paola De Luca', Geraldine Gueron², Alejandra Paez², \\ Cristian P. Moiola ${ }^{1}$, Cintia Massillo', Juliana Porretti ${ }^{1}$, Jimena Giudice ${ }^{3}$, Florencia Zalazar ${ }^{1}$, \\ Nora Navone ${ }^{4}$, Elba Vazquez ${ }^{2}$, and Adriana De Siervi ${ }^{1}$
}

\section{Abstract}

Prostate cancer is the second leading cause of cancer-related death in men worldwide. Many factors that participate in the development of prostate cancer promote imbalance in the redox state of the cell. Accumulation of reactive oxygen species causes injury to cell structures, ultimately leading to cancer development. The antioxidant enzyme heme oxygenase 1 (HMOX1/HO-1) is responsible for the maintenance of the cellular homeostasis, playing a critical role in the oxidative stress and the regulation of prostate cancer development and progression. In the present study, the transcriptional regulation of $\mathrm{HO}-1$ was investigated in prostate cancer. Interestingly, the tumor suppressor BRCA1 binds to the HO-1 promoter and modulates $\mathrm{HO}-1$, inducing its protein levels through both the increment of its promoter activity and the induction of its transcriptional activation. In addition, in vitro and in vivo analyses show that BRCA1 also controls HO-1-negative targets: MMP9, uPA, and Cyclin D1. HO-1 transcriptional regu- lation is also modulated by oxidative and genotoxic agents Induction of DNA damage by mitoxantrone and etoposide repressed HO-1 transcription, whereas hydrogen peroxide and doxorubicin induced its expression. Xenograft studies showed that HO-1 regulation by doxorubicin also occurs in vivo. Immunofluorescence analysis revealed that BRCA1 overexpression and/or doxorubicin exposure induced the cytoplasmic retention of HO-1. Finally, the transcription factor NRF2 cooperates with BRCA1 protein to activate $\mathrm{HO}-1$ promoter activity. In summary, these results show that the activation of BRCA1-NRF2/HO-1 axis defines a new mechanism for the maintenance of the cellular homeostasis in prostate cancer.

Implications: Oxidative and genotoxic stress converge on HO-1 transcriptional activity through the combined actions of BRCA1 and NRF2. Mol Cancer Res; 13(11); 1455-64. @2015 AACR.

\section{Introduction}

Prostate cancer is one of the most common cancers and the second leading cause of cancer-related death in men worldwide (1). Both environmental and genetic factors are involved in its etiology, turning prostate cancer into a heterogeneous disease and thus making it extremely difficult to design effective therapeutic strategies (2). In particular, inflammation, triggered by infectious

\footnotetext{
'Laboratorio de Oncología Molecular y Nuevos Blancos Terapéuticos, Instituto de Biologia y Medicina Experimental (IBYME)-CONICET, Buenos Aires, Argentina. ${ }^{2}$ Laboratorio de Inflamación y Cáncer, Departamento de Química Biológica, Facultad de Ciencias Exactas y Naturales (FCEN), Universidad de Buenos Aires (UBA), IQUIBICEN-CONICET, Buenos Aires, Argentina. ${ }^{3}$ Department of Pathology and Immunology, Baylor College of Medicine, Houston, Texas. ${ }^{4}$ Department of Genitourinary Medical Oncology, The University of Texas, M. D. Anderson Cancer Center, Houston, Texas.

Note: Supplementary data for this article are available at Molecular Cancer Research Online (http://mcr.aacrjournals.org/).

Corresponding Author: Adriana De Siervi, Instituto de Biologia y Medicina Experimental (IBYME)-CONICET, Vuelta de Obligado 2490, Buenos Aires C1428ADN, Argentina. Phone: 5411-4783-2869; Fax: 5411-4786-2564; E-mail: adrianadesiervi@gmail.com

doi: 10.1158/1541-7786.MCR-15-0150-T

(C)2015 American Association for Cancer Research.
}

agents or exposure to environmental factors, increases prostate cancer tumorigenesis (3).

Oxidative stress represents an unbalanced situation between pro-oxidative factors and oxidants controlled by multiple components that can cause cellular damage. Reactive oxygen species (ROS), produced during metabolism, are decisive to oxidative stress (4). Once accumulated within the cell, ROS can damage proteins, lipids, and DNA, contributing to a variety of human pathogenesis, including cardiovascular, metabolic, inflammatory, neurodegenerative diseases, and cancer (5). ROS also act as second messengers in cell signaling being involved in a wide range of cellular processes, such as proliferation, cell-cycle arrest, and cell death $(6,7)$.

The key to understand the molecular mechanisms underlying redox regulation vests in characterizing the regulatory molecules mediating ROS intracellular homeostasis. Tumor-suppressor genes possess protective functions that limit tumor growth and regulate many cellular functions (8). BRCA1 is a tumorsuppressor gene that participates in DNA-damage repair, cell-cycle regulation, maintenance of genomic integrity, and is involved in numerous pathways as a transcriptional coregulator. Some of these functions, mainly its participation in DNA-damage response, could be associated to an essential participation of BRCA1 in oxidative stress response. It was previously demonstrated that BRCA1 overexpression provides resistance to different oxidant agents and that BRCA1 induces antioxidant transcription factor 
NRF2 (NF-E2-related factor 2) activity, suggesting a novel role for BRCA1 in protection against oxidative stress (9). Furthermore, BRCA1 together with NRF2 preserve cells against oxidative stress by coregulating genes containing antioxidant response elements (ARE) on their promoter regions. In addition, through a chromatin immunoprecipitation analysis combined with microarray technology (ChIP-chip) and gene expression studies, we have previously described that BRCA1 is a transcriptional coregulator of genes involved in the DNA-damage response (10-12).

Heme Oxygenase 1 (HO-1), generally localized in the cytoplasm, is the rate-limiting enzyme in heme degradation, a powerful pro-oxidative and proinflammatory agent (13). In this context, HO-1 anti-inflammatory and antioxidative effects provide cells, tissues, and organs protection from environmental variations. Thus, HO-1-deficient mice are more sensitive to free radical accumulation $(14,15)$, and humans lacking HO-1 present severe endothelial damage and sustained inflammation (16). Previous studies from our laboratory showed that HO-1 is implicated in prostate cancer, unveiling that endogenous $\mathrm{HO}-1$ inhibits bone-derived prostate cancer cell proliferation, invasion, and migration in vitro and decreases tumor growth and angiogenesis in vivo $(14,17)$, suggesting its antitumoral function. Despite its importance in cellular homeostasis, the molecular mechanisms underlying the transcriptional regulation of $\mathrm{HO}-1$ are still poorly understood (18).

In this study, we provide an unprecedented mechanism that regulates HO- 1 transcription. We demonstrated that BRCA1 regulates HO-1 expression in prostate cancer cells. Furthermore, we determine that different oxidative stress or DNA-damage agents, such as mitoxantrone and etoposide, impair HO-1 transcription. This study describes a novel mechanism for HO-1 transcriptional regulation mediated by BRCA1, shedding light into its role in the maintenance of the cellular homeostasis.

\section{Materials and Methods}

\section{Cell culture and treatments}

PC3 (ATCC: CRL-1435), LNCaP (ATCC: CRL-1740), 22Rv1 (ATCC: CRL-2505), and C4-2 (derived from LNCaP) cells were routinely cultured in RPMI 1640 (Invitrogen) supplemented with 10\% FBS. PC3 stable cell lines (pcDNA3, pcDNA3 BRCA1, shRNA Scramble, and shRNA BRCA1) and LNCaP stable cell lines (shRNA Scramble and shRNA BRCA1) were previously described $(11,19)$. Doxorubicin (Rontag S.A.) was used at a final concentration of $1 \mu \mathrm{mol} / \mathrm{L}$ and $2 \mu \mathrm{mol} / \mathrm{L}$ for $0,6,18$, and 24 hours. Mitoxantrone (Micraleve; Ivax, Argentina) was used at final concentration of $1 \mu \mathrm{mol} / \mathrm{L}$ for 24 hours. Etoposide (Sigma-Aldrich) was used at final concentration of $5 \mu \mathrm{mol} / \mathrm{L}$ for 24 hours. Methotrexate (Ervemin, Ivax, Argentina) was used at final concentration of $200 \mu \mathrm{mol} / \mathrm{L}$ for 24 hours. Hydrogen peroxide (ParaFarm, Saporiti, Argentina) was used at final concentration of $400 \mu \mathrm{mol} / \mathrm{L}$ for 6 hours.

\section{Plasmids}

BRCA1 expression vector (pcDNA3 BRCA1) has been previously described in ref. 20. shRNA Scramble and shRNA BRCA1 vectors were from Upstate. hHO4.9-luc (HO1-luc) and hHO4.9_M2-luc (mutHO1-luc) were previously described (17). pCDNA3 NRF2 and pcDNA3 NRF2 dominant-negative (DN) vectors were kindly provided by Dr. Omar Coso (IFIBYNE, University of Buenos Aires, Argentina).

\section{Immunoblot analysis}

Prostate cancer cells were lysed and immunoblotted as previously described (17) using antibodies against HO-1 (Stressgen Biotechnologies, Corp.), $\beta$-actin (Santa Cruz Biotechnology), and BRCA1 protein, gently provided by Dr. Kevin Gardner (NCI-NIH; ref. 10). Reactions were detected by horseradish peroxidaseconjugated secondary antibodies and enhanced chemiluminescence (Pierce) following the manufacturer's directions. Protein quantitation was performed using Image J 1.41 software (http:// rsb.info.nih.gov, NIH).

\section{Luciferase reporter assay}

PC3 cells were transiently transfected by the polyethylenimine method with $1 \mu \mathrm{g}$ of each plasmid. After transfection cells were maintained in complete medium for 48 hours, they were harvested and lysed on Reporter Lysis Buffer with $40 \mu \mathrm{L}$ of Steady Glomax Luciferase System (Promega). Luciferase activity was determined in Luminometer (Glomax Multi Detection System; Promega), and data were normalized to total protein determined by the Bradford assay.

\section{Human prostate cancer xenografts}

Xenograft experiments were performed as previously described (11). All animal experiments were conducted in accordance with accepted standards of animal care and were approved by the Institutional Animal Care and Use Committee (IACUC) of the University of Buenos Aires (http://users.df.uba.ar/zeke/). Briefly, 6-week-old male nu/nu mice, each weighting at least $20 \mathrm{~g}$, were housed under pathogen-free conditions. Mice were randomized into two groups: shRNA Scramble or shRNA BRCA1. Tumor cells $\left(4.8 \times 10^{6}\right.$ cells $)$ were inoculated s.c. into the right flank of the mice. After 14 days, when the tumors reached approximately 50 $\mathrm{mm}^{3}$, mice were randomly distributed into two groups ( 5 mice per group) and injected i.p. with doxorubicin ( $8 \mathrm{mg} / \mathrm{kg}$ mouse). A second injection was administered after 9 days, and the mice were sacrificed 24 hours after the last injection. RNA was isolated from tumors as described (17).

\section{RNA isolation, cDNA synthesis, and quantitative PCR}

Prostate cancer cells were exposed to different treatments, and total RNA was isolated using TriReagent (Genbiotech). cDNA was retrotranscribed from $2 \mu \mathrm{g}$ of RNA using RevertAid First Strand (Fermentas). Real-time PCR (qPCR) was performed as previously described (21) using Taq Polymerase (Invitrogen) and SYBR Green as fluorescent dye for amplified product detection in a DNA Engine Opticon (MJ Research, BioRad). Each PCR was done in duplicate, and the experiment was repeated at least thrice. Data were analyzed by Opticon- 3 software and normalized to $\beta$-actin (ACTB). Errors were calculated as previously described (10). Primers sequences were previously described: BRCA1 (10), HO-1, MMP9 (17), uPA, Cyclin D1, and АCTB (21).

\section{Chromatin immunoprecipitation}

Chromatin immunoprecipitation (ChIP) was performed from PC3 cells as previously described (12) using anti-BRCA1 (10) antibodies. Anti-Gal4 antibody from Santa Cruz Biotechnologies was used as nonspecific control. ChIP-DNA was amplified by qPCR using primers mapping at $9 \mathrm{~Kb}$ and $4 \mathrm{~Kb}$ upstream from HO-1 transcription start site (TSS), or $0.2 \mathrm{~Kb}$ upstream from $\beta$-globin. Primer sequences were as follows: ChIP HO-1 -9 kb 
FW 5'CCCTGCTGAGTAATCCTTTCCCGA3' and RV 5'ATGTCCCGACTCCAGACTCCA3'; ChIP HO- $1-4$ kb FW 5'GGTAGGCAGGAGGAAGTG3' and RV 5'AGAGATGTGAAGCATAAGAAGG3'; $\beta$-globin FW 5'TTTGCAGCCTCACCTTCTTT3' and RV 5'TGGGGGATATTATGAAGGGC3'. Fold enrichment was calculated normalizing data to input as previously described (12).

\section{Immunofluorescence experiments}

Immunofluorescence experiments were performed as previously described (21). Briefly, cells were seeded in 6-well plate at a density of $1 \times 10^{6}$ cells per well on coverslips overnight. After treatments, cells were fixed in ice-cold methanol, permeabilized with $0.5 \%$ Triton X-100/PBS, washed, and blocked. Cells were incubated overnight with primary antibodies and after 3 to 4 washes were incubated with fluorescent secondary antibodies. Negative controls were carried out using PBS instead of primary antibodies. Cells were washed, mounted in FluorSave Reagent (Merck Millipore), and imaged by confocal laser scanning microscopy with an Olympus Fluoview FV 1000 microscope. An Olympus $60 \times 1.20$ N.A. UPLAN APO water immersion objective was used. Excitation and emission filter for Alexa Fluor-647 were as follow: excitation, $650 \mathrm{~nm}$; emission, band-pass (BP) $665 \mathrm{~nm}$.

All the images were processed for presentation applying exactly the same parameters using ImageJ (http://rsb.info.nih.gov, NIH). Background of each channel was subtracted, a median filter (radius: 1 pixel) was applied. Quantitative microscopy measurements were performed in individual cells using ImageJ (http://rsb. info.nih.gov, $\mathrm{NIH}$ ) as previously described (21-23). Briefly, the signal of the secondary antibody alone was subtracted for all the HO-1 images. Segmentation was manually performed for each cell using the HO-1 (green) images, and HO-1 signal ("HO-1 cell") was measured as well as the cell size; therefore, HO-1 fluorescence was normalized to the cell size. The nucleus was segmented using DAPI signal (blue), and HO-1 signal was measured within this region obtaining "HO-1 nucleus." The ratio "HO-1 cell"/"HO-1 nucleus" was then calculated as an estimation of HO-1 intracellular distribution. This "internal calibration" approach removes the influence of the amplifier gain and the zoom factor for each image acquisition condition (24). Results are shown as mean $( \pm)$ SEM of " $n$ " cells.

\section{Statistical analysis}

All results are given as mean $( \pm)$ SD of " $n$ " separate independent experiments unless stated otherwise. Student tests were used to ascertain statistical significance with a threshold of $P<0.05$. Comparisons for in vivo experiments were made with one-way ANOVA followed by the Dunnett test, with $P<0.05$ as the criterion for statistical significance.

\section{Results}

Prostate cancer cells express different levels of HO-1

We first assessed BRCA1 and HO-1 expression levels in different human prostate cancer cell lines: PC3 (androgen receptor negative), LNCaP (androgen receptor positive), 22Rv1 (androgen receptor positive), and C4-2 (androgen receptor positive, derived from LNCaP after grown in castrated mice, androgen insensitive). LNCaP cells showed the highest levels of HO-1 and BRCA1, whereas the lowest expression for both proteins was found in PC3 cells (Fig. 1A). As we previously reported, HO-1 expression was higher in androgen-sensitive cell lines (17).
BRCA1 induces HO-1 transcription in prostate cancer cells

We previously reported that BRCA1 induces the expression of genes involved in stress response and resistance to genotoxic agents in prostate cancer $(10-12,19)$. In addition, it is known that BRCA1 protects cells against oxidative stress upregulating genes carrying ARE (9). Given that HO-1 contains ARE sites in its promoter region and participates in the maintenance of the cellular homeostasis by reducing oxidative injury, we investigated whether BRCA1 could regulate $\mathrm{HO}-1$ expression in prostate cancer cell lines.

We generated BRCA1-overexpressing or depleted stable-transfected PC3 cell lines and BRCA1-depleted stable LNCaP cells and assessed HO-1 protein and mRNA levels (Fig. 1B-D). BRCA1overexpressing PC3 cells displayed increased $\mathrm{HO}-1$ protein and mRNA levels compared with control cells (Fig. 1B and C). Consistently, HO-1 induction was abolished after BRCA1 depletion by shRNA in PC3 and LNCaP cells (Fig. 1C and D). Furthermore, we determined HO-1 expression in BRCA1-overexpressing transiently transfected C4-2 and 22v1 cells by RT-qPCR. HO-1 mRNA levels were also induced by BRCA1 overexpression in all cell lines tested (Fig. 1B). Given that BRCA1 regulates HO-1 expression in all the prostate cancer cell lines tested, we choose PC3 cells for the following experiments performed.

To elucidate whether BRCA1 regulated $\mathrm{HO}-1$ promoter activity, we carried out reporter assays by cotransfecting PC3 cells with BRCA1 expression or control vectors and a construct containing the $4.9 \mathrm{~kb}$ promoter region of the human wild-type HO-1 gene upstream of the luciferase reporter gene (HO1-luc; ref. 17). HO-1 luciferase reporter assays showed that BRCA1 overexpression significantly induced $\mathrm{HO}-1$ promoter activity in PC3 cells (Fig. 1E). Altogether, these results demonstrate that BRCA1 regulates HO-1 expression at the transcription level in prostate cancer cell lines.

\section{BRCA1 protein binds the HO-1 promoter}

To further investigate HO-1 regulation by BRCA1, we performed BRCA1 ChIP in PC3 cells and found that BRCA1 binds the HO-1 promoter at $4 \mathrm{~Kb}$ and $9 \mathrm{~Kb}$ upstream of the HO-1 TSS (Fig. 1F). These results show that BRCA1 associates with HO-1 promoter in PC3 cells.

BRCA1 switches off negative targets of $\mathrm{HO}-1$ in prostate cancer

We have previously shown that HO-1 has the ability to associate with promoters of genes relevant to prostate carcinogenesis, such as $u P A, M M P 9$, and cyclin D1 (21). Moreover, HO-1 induction in prostate cancer impairs MMP9 expression and activity (17). To investigate whether BRCA1 modulates HO-1 function, we assessed the mRNA levels of HO-1 target genes under BRCA1 modulation. BRCA1 overexpression in PC3 cells turned off downstream targets of HO-1: MMP9, uPA, and Cyclin D1 (Fig. 2A). Accordingly, uPA and Cyclin D1 expression levels were induced in BRCA1-depleted PC3 cells (Fig. 2B). No significant changes were observed at MMP9 expression levels after BRCA1 depletion (Fig. 2B)

Furthermore, we developed a xenograft model in $n u / n u$ mice by injection of BRCA1-depleted PC3 (shRNA BRCA1) or control cells (shRNA Scramble; ref. 11). We previously demonstrated that BRCA1 depletion increased tumor growth rate (11). To determine whether BRCA1 modulates HO-1 expression and function in vivo, we analyzed HO-1 and the expression levels of its target genes by RT-qPCR. Even though no significant differences were detected in 
A

\section{PC3 LNCaP 22Rv1 C4-2}

$\mathrm{HO}-1$

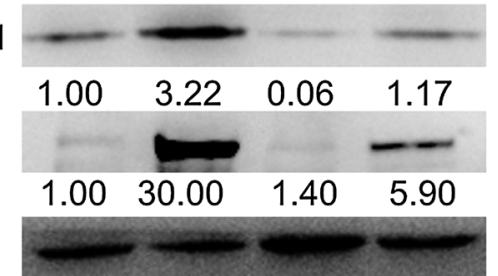

B BRCA1 overexpression

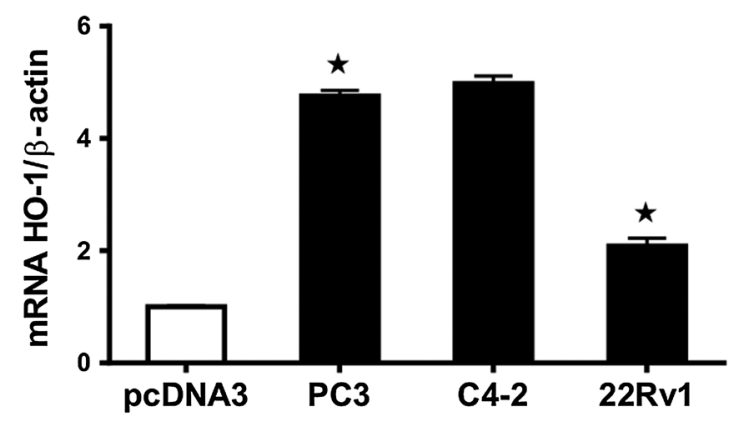

D BRCA1 depletion
C

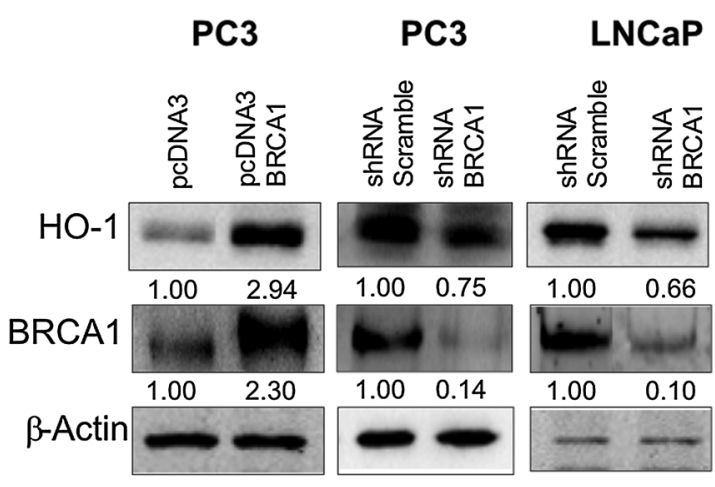

E

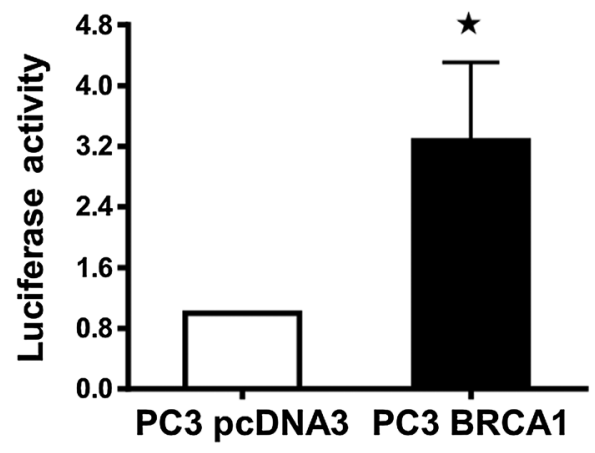

F $\quad$ BRCA1 ChIP

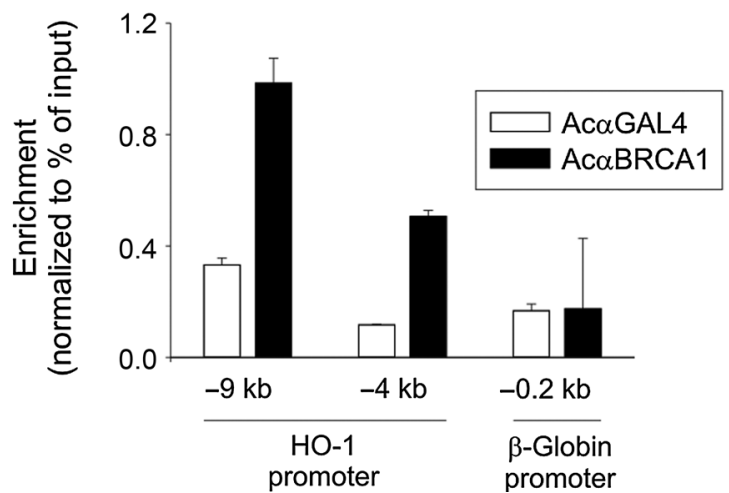

Figure 1.

BRCA1 upregulates HO-1 expression in prostate cancer cell lines. A, HO-1, BRCA1, and $\beta$-actin expression was determined in PC3, LNCaP, 22Rv1, and C4-2 cell lines by Western blot. Quantification and normalization to $\beta$-actin protein levels and PC3 cells are shown under each band. One representative experiment from two biologic replicates is shown. B, HO-1 mRNA levels were analyzed by RT-qPCR in PC3, C4-2, and 22Rv1 cells transfected with pcDNA3 BRCA1 plasmid or control (pcDNA3). Data were normalized to $\beta$-actin and pcDNA3-transfected cells. One representative from two independent experiments is shown $\left({ }^{*}, P<0.05\right)$. C, PC3 (pcDNA3, pcDNA3 BRCA1, shRNA Scramble, and shRNA BRCA1) and LNCaP (shRNA Scramble and shRNA BRCA1) stable cell lines were generated, and $\mathrm{HO}-1, \mathrm{BRCA} 1$, and $\beta$-actin expression was determined by Western blot. Numbers below bands show quantitation using ImageJ software. Data were normalized to $\beta$-actin and control cells. One representative of two independent experiments is shown. D, HO-1 mRNA levels were analyzed by RT-qPCR in LNCaP shRNA Scramble and LNCaP shRNA BRCA1 stable cells lines. Data were normalized to $\beta$-actin and control cells. One representative from two independent experiments is shown $\left({ }^{*}, P<0.05\right)$. E, reporter assay in PC3 cells transfected with HO- 1 luciferase plasmid. After transfection, cells were maintained in complete medium, lysed, and luciferase activity was assayed. Data were normalized to protein values and control cells. Mean \pm SD from three biologic replicates is shown $\left(^{*}, P<0.05\right)$. F, ChIP analysis using BRCA1-specific or non-specific (GAL4) antibodies in PC3 cells was performed. DNA-ChIP was analyzed by qPCR using primers located at $9 \mathrm{~Kb}$ and $4 \mathrm{~Kb}$ upstream from HO-1 TSS or $0.2 \mathrm{~Kb}$ upstream from $\beta$-globin TSS.

Fold enrichment was calculated normalizing data to input. 
A BRCA1 overexpression
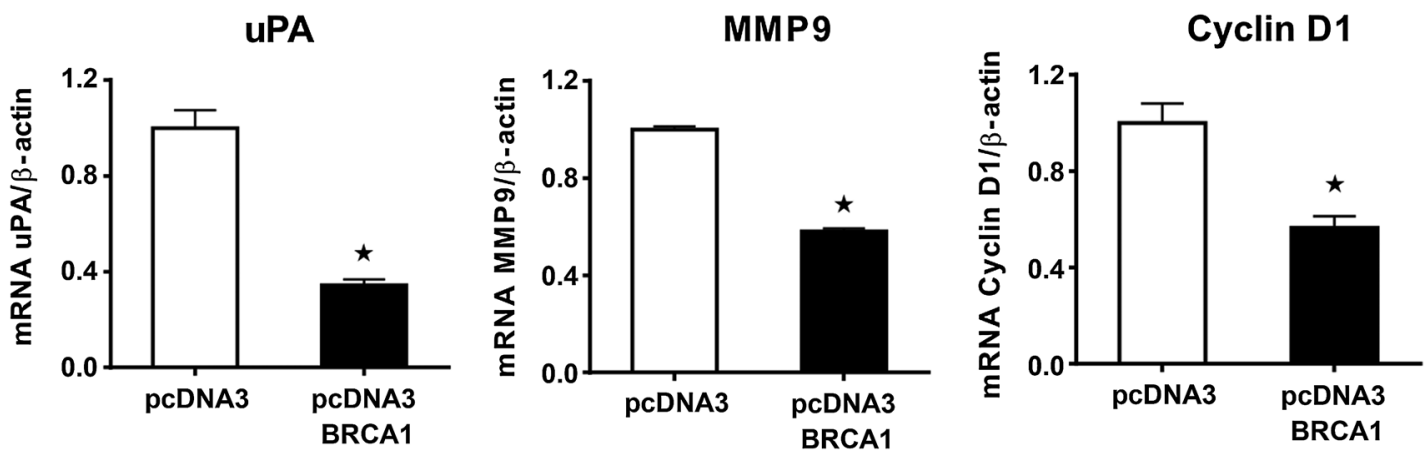

B BRCA1 depletion
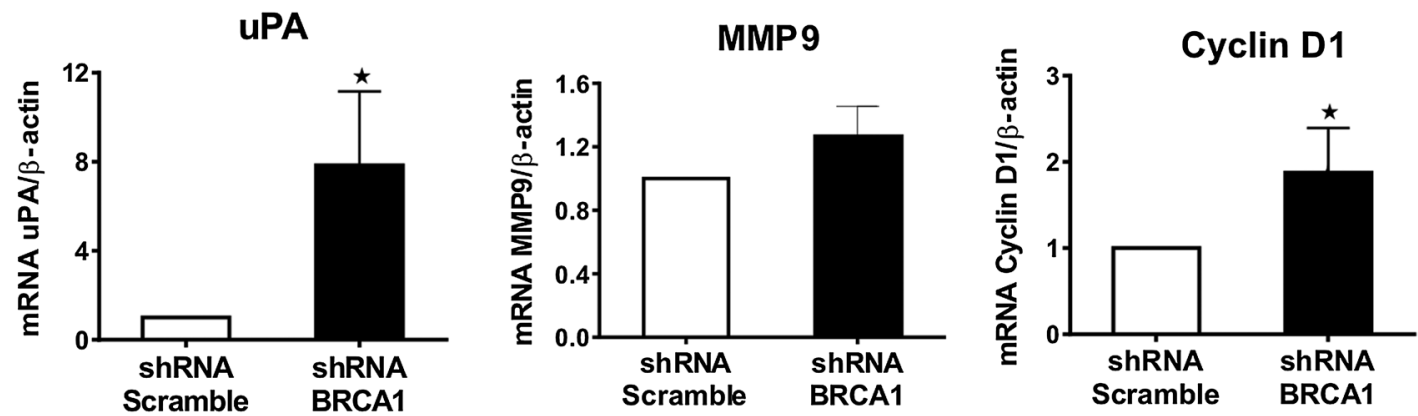

C Xenografts

HO-1

UPA
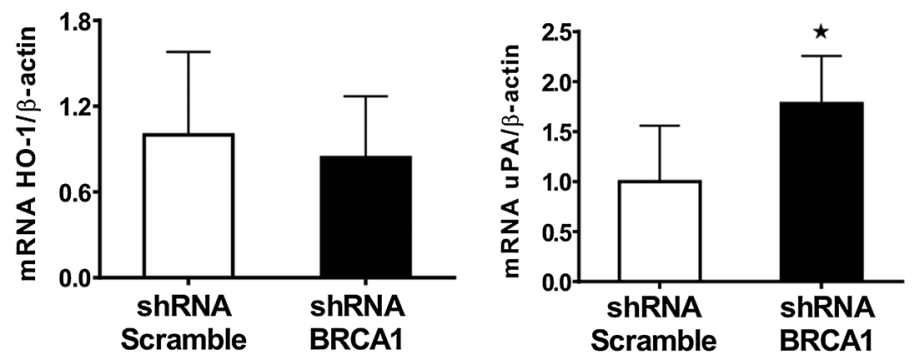

MMP9

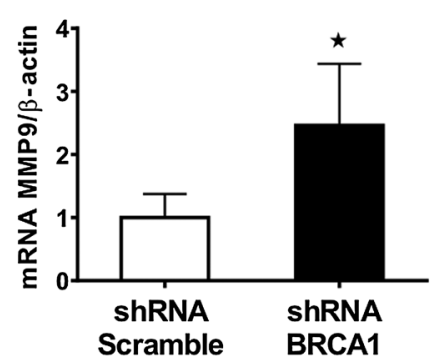

Cyclin D1

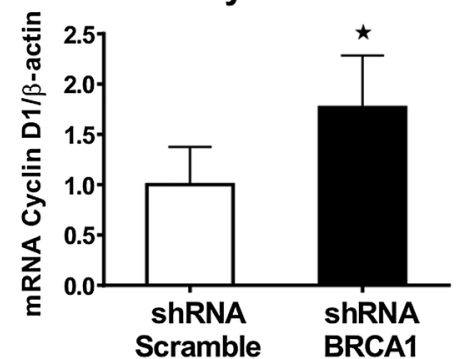

Figure 2.

BRCA1 represses HO-1-negative target genes in prostate cancer. A, mRNA expression levels for the indicated genes were analyzed by RT-qPCR in PC3 pcDNA3 and pcDNA3 BRCA1 stable cell lines. Data were normalized to $\beta$-actin and control. One representative experiment from three biologic replicates is shown $\left({ }^{*}, P<0.05\right)$. B, mRNA expression levels for the indicated genes were analyzed by RT-qPCR in PC3 shRNA Scramble and shRNA BRCA1 stable cell lines. Data were normalized to $\beta$-actin and control. One representative experiment from three biologic replicates is shown $\left(^{*}, P<0.05\right)$. $C$, harvested tumors derived from PC3 shRNA Scramble and shRNA BRCA1 cells were snap-frozen in liquid nitrogen and subsequently processed for RNA isolation. mRNA expression levels for the indicated genes were analyzed by RT-qPCR. Data were normalized to $\beta$-actin and control. Bars represent mean \pm SD from five biologic independent xenografts $\left({ }^{*}, P<0.05\right)$. 
A

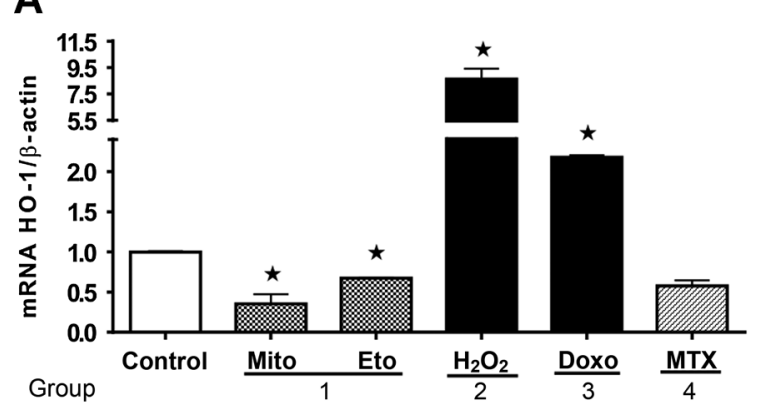

B

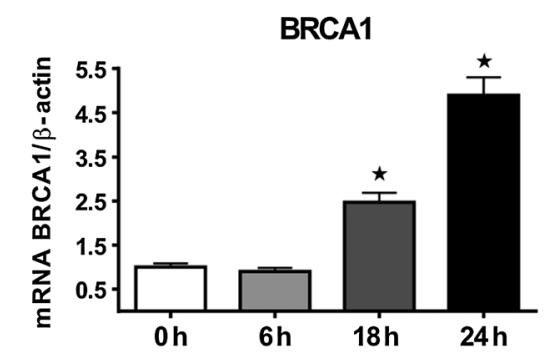

C

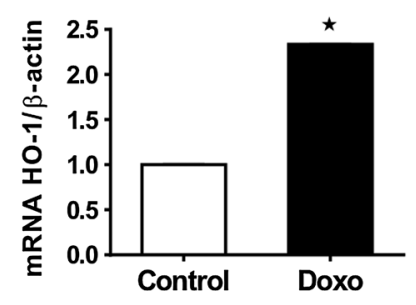

Figure 3.

HO-1 transcription is modulated in response to genotoxic and cytotoxic agents. A, HO-1 expression was analyzed by RT-qPCR after PC3 cells exposure to: group 1 = DNA-damage agents: mitoxantrone (Mito: $1 \mu \mathrm{mol} / \mathrm{L}$ 24 hours) and etoposide (Eto: $5 \mu \mathrm{mol} / \mathrm{L}, 24$ hours); group

2 = oxidative stress agents: hydrogen peroxide $\left(\mathrm{H}_{2} \mathrm{O}_{2}: 400 \mu \mathrm{mol} / \mathrm{L}, 6\right.$ hours $)$ group $3=$ combined effects agents: doxorubicin (Doxo: $2 \mu \mathrm{mol} / \mathrm{L}$, 24 hours); group $4=$ other effects agents: methotrexate (MTX: $200 \mu \mathrm{mol} / \mathrm{L}, 24$ hours). Data were normalized to $\beta$-actin and vehicle (DMSO). One representative from two independent experiments is shown $\left({ }^{*}, P<0.05\right)$. B, BRCA1 and HO-1 RT-qPCR from PC3 cells treated with doxorubicin $(1 \mu \mathrm{mol} / \mathrm{L})$ for the indicated times (0 to 24 hours). Data were normalized to $\beta$-actin and control (DMSO). One representative from three independent experiments is shown $\left(^{*}, P<0.05\right)$. C, prostate cancer xenografts derived from PC3 cells were grown in nude mice. Mice were injected i.p. with doxorubicin (Doxo; $8 \mathrm{mg}$ doxorubicin/kg mouse) or vehicle (control; DMSO) on days 14 and 24. Mice were sacrificed 24 hours after the last injection, and RT-qPCR analysis of HO-1 was carried out. Graph bar represents the mean \pm SD from five tumors. Data were normalized to $\beta$-actin and control $\left({ }^{*}, P<0.05\right)$.
HO-1 expression levels, BRCA1-depleted tumors showed highly increased expression of MMP9, uPA, and Cyclin D1 (Fig. 2C). Altogether, our results show that BRCA1 reduces the expression levels of HO-1 downstream targets both in vitro and in vivo.

Genotoxic and cytotoxic agents modulate $\mathrm{HO}-1$ transcription BRCA1 participates in the DNA-damage response caused by genotoxic agents through specific gene-target regulation (11). To assess the involvement of the BRCA1/HO-1 axis in the oxidative stress response in prostate cancer, we assessed HO-1 regulation in response to a variety of genotoxic and oxidizing agents. HO-1 levels were determined by RT-qPCR after exposure of PC3 cells to a panel of drugs: group 1, DNA-damage inducers: mitoxantrone and etoposide; group 2, oxidative stress inducers: hydrogen peroxide; group 3, DNA-damage/oxidative stress inducers: doxorubicin; and group 4, other effect inducers: methotrexate. The results displayed in Fig. 3A show that HO-1 transcription was significantly downregulated by group 1 agents, upregulated by groups 2 and 3 agents, whereas no significant variation was observed for group 4 drugs.

We subsequently focused our studies in the effect of doxorubicin on HO-1 transcription. Previous findings of our laboratory demonstrated that this drug upregulates BRCA1 expression (10). We performed a time-response treatment by exposing PC3 cells to this agent and then determined HO- 1 and BRCA1 mRNA levels using RT-qPCR. We found that HO- 1 and BRCA1 expres- sion levels were induced after 18-hour doxorubicin treatment (Fig. 3B).

To examine HO-1 modulation after doxorubicin treatment in vivo, $n u / n u$ mice were injected s.c. with PC3 cells and, 14 days after injection, mice were randomly distributed into two groups (5 mice per group) and administrated i.p. doxorubicin or vehicle. Tumors were excised, and RT-qPCR analysis revealed a significant induction of HO-1 transcription in doxorubicin-treated xenografts (Fig. 3C). These results demonstrate that doxorubicin induces HO-1 expression in vivo.

BRCA1 and/or doxorubicin increase HO-1 expression and cytoplasmic retention

Since nuclear localization of HO-1 has been reported in prostate cancer $(17,25,26)$, we investigated BRCA1 and/or doxorubicin effects on HO-1 subcellular localization by immunofluorescence analysis.

Although some HO-1 nuclear signal was found in PC3 cells, it was mainly detected in the cytoplasmic compartment (Fig. 4A, control cells). HO-1 nuclear signal/HO-1 total signal ratio was measured in individual cells (Fig. 4B), revealing that BRCA1 overexpression induces HO-1 cytoplasmic retention (Fig. 4A and C).

Doxorubicin treatment also causes HO-1 retention in the cytoplasm of control cells as well as under BRCA1-induced conditions (Figs. 4A and C). 
A
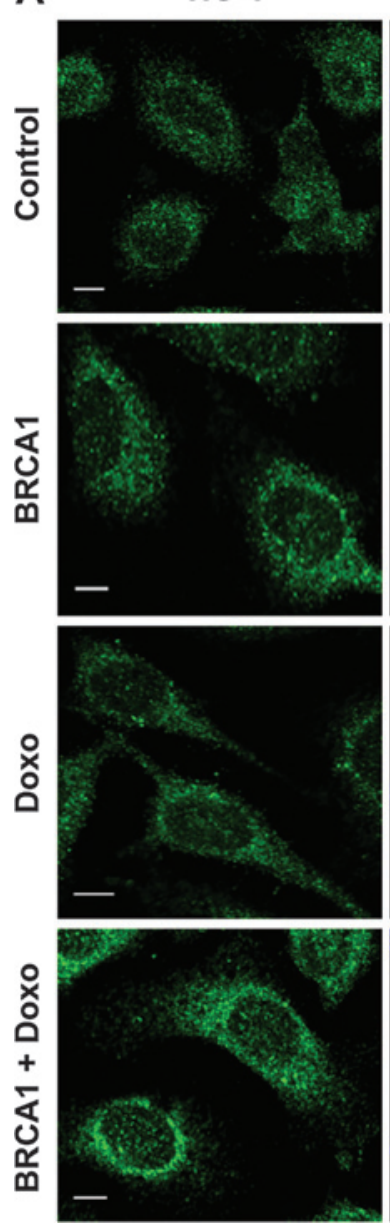

DAPI
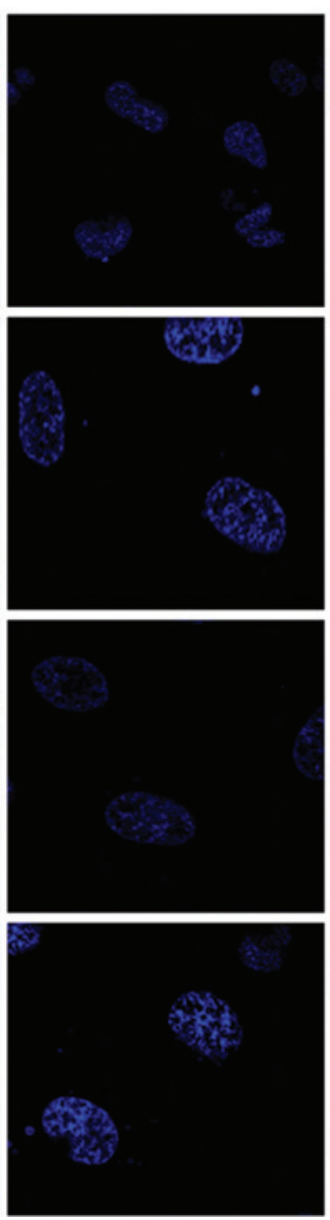

Overlay
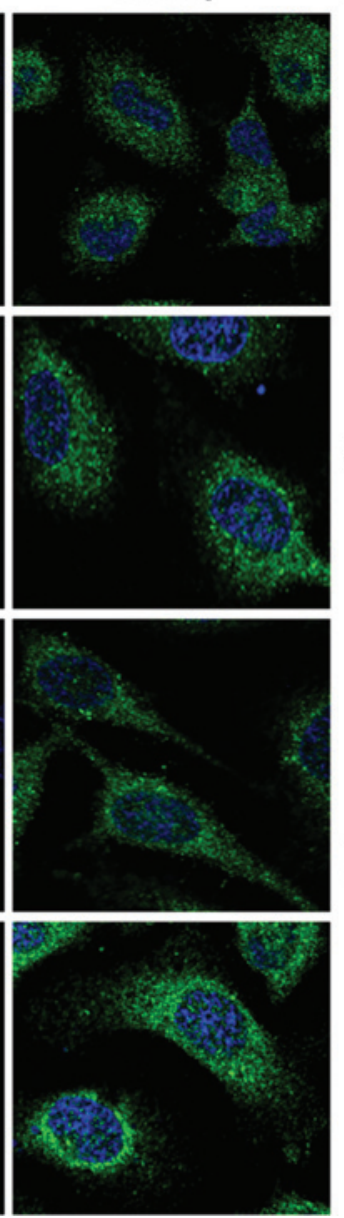

B

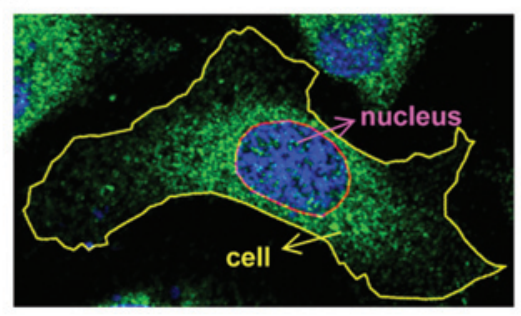

Nucleus $/$ total $=\frac{\text { Signal HO-1 nucleus }}{\text { Signal HO-1 cell }}$

C
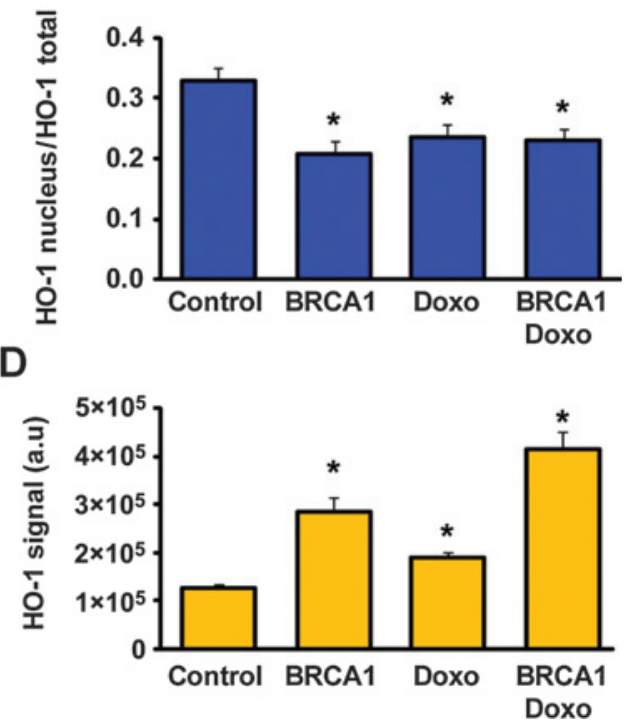

Figure 4.

BRCA1 overexpression and/or doxorubicin treatment induces HO-1 expression and favors its cytoplasmic localization in PC3 cells. A, PC3 cells transiently transfected with BRCA1 overexpression or control empty vector were treated with doxorubicin (Doxo; $2 \mu \mathrm{mol} / \mathrm{L}, 24 \mathrm{hours}$ ) or vehicle. Cells were fixed in methanol, and HO-1 distribution was analyzed by confocal fluorescence microscopy (green: Alexa Fluor 647). Nuclei were stained using DAPI (blue). B, cell outline and whole-cell nuclear segmentation to calculate nuclear $\mathrm{HO}-1 /$ total $\mathrm{HO}-1$ ratio in a single-cell analysis. $\mathrm{C}$, quantification of nuclear to cellular $\mathrm{HO}-1$ signal relation $\left(^{*}, P<0.05\right.$, respect to control). D, total HO-1 signal quantification $\left(^{*}, P<0.05\right.$, respect to control).

In addition, quantification of HO-1 total signal confirms that BRCA1 overexpression results in a significant increase in HO-1 levels, which are even significantly higher when cells are exposed to doxorubicin (Fig. 4D).

BRCA1 modulates $\mathrm{HO}-1$ transcription through coregulation with NRF2

It has long been known that the transcription factor NRF2 is a transcription regulator of HO-1 (18). Thus, we hypothesized that BRCA1 protein and NRF2 could coregulate HO-1 transcription. For this purpose, we assessed HO-1 mRNA levels in PC3 cells after NRF2 modulation. NRF2 overexpression significantly induced HO-1 mRNA levels and promoter activity in these cells (Fig. 5A and $\mathrm{B}$ ). We previously demonstrated that BRCA1 regulates gene expression through the direct interaction with transcription factors $(10,11)$. Considering that both, BRCA1 and NRF2, are involved in HO-1 regulation, we assessed the HO-1 transcriptional coregulation by these factors using reporter assays. As shown in Fig. 5C, BRCA1 and NRF2 overexpression resulted in a higher activation of the HO-1 promoter, compared with either of them alone.

Consistently, enhanced HO-1 promoter activity by BRCA1 overexpression was lost when the NRF2 DN construct was cotransfected together with BRCA1 expression vector and HO1-luc reporter plasmid (Fig. 5C). These results provide evidence that HO-1 transcription activation by BRCA1 is NRF2 dependent.

Furthermore, we performed reporter assays using an HO-1 promoter construct carrying a mutation in the MARE site (Maf recognition element site; mutHO1-luc). HO-1 promoter activity induction by BRCA1, NRF2, and the combination of both factors was completely abolished when the MARE site at $\mathrm{HO}-1$ promoter was mutated (Fig. 5C). These results suggest that antioxidant response elements are required for HO-1 modulation by BRCA1 and NRF2.

Finally, we analyzed HO-1 promoter activity after BRCA1 depletion (Fig. 5D). We found that BRCA1 depletion significantly reduced HO-1 promoter activity. Interestingly, BRCA1 depletion completely abrogated transcriptional induction of HO- 1 by NRF2 
Labanca et al.

A
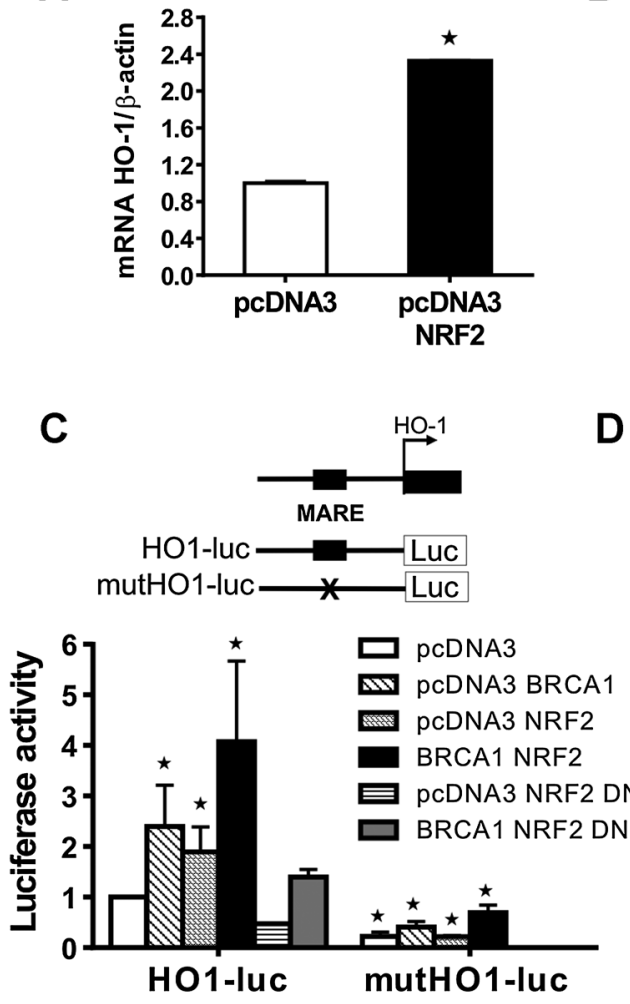

B

D
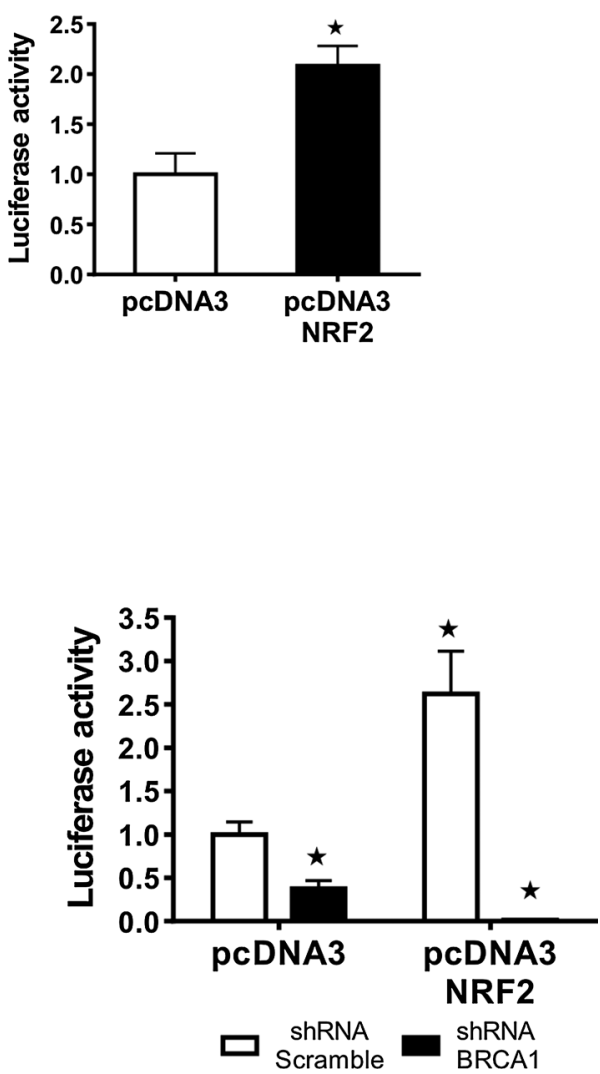

Figure 5.

BRCA1, together with NRF2, induces HO-1 promoter activity in prostate cancer cells, and MARE site in HO-1 promoter is required for this regulation. A, HO-1 mRNA levels were analyzed by RT-qPCR in PC3 pcDNA3 and PC3 pcDNA3 NRF2 transient transfected cells. Data were normalized to $\beta$-actin and control. One representative from at least three independent experiments is shown $\left(^{*}, P<0.05\right)$. B, PC3 cells were cotransfected with the HO- 1 luciferase plasmid and pCDNA3 NRF2 or empty vector (pcDNA3). Cells were maintained in complete medium and 48 hours after transfection were lysed, and luciferase activity was assayed. Data were normalized to protein values and control. One representative from at least three independent experiments is shown (*, $P<0.05$ ). $C$, PC3 transiently expressing HO1-luc reporter plasmid or the variant containing the MARE site mutated was cotransfected with BRCA1 overexpression vector (pcDNA3 BRCA1) and NRF2 overexpression vector (pcDNA3 NRF2) or NRF2 DN vector (pcDNA3 NRF2 DN) or control empty vector (pcDNA3). Cells were harvested 48 hours after transfection, and luciferase activity was measured. Data were normalized to protein values and control. Mean \pm SD from three biologic independent experiments is shown $\left.{ }^{*}, P<0.05\right)$. D, PC3 cells were cotransfected with HO1-luc reporter plasmid and pcDNA3 NRF2 or pcDNA3 empty vector, and shRNA BRCA1 or shRNA Scramble vectors. Cells were harvested 48 hours after transfection, and luciferase activity was measured. Transfections were performed in triplicate in at least three biologic independent experiments $\left({ }^{*}, P<0.05\right)$.

(Fig. 5D). Altogether, these results suggest that BRCA1 and NRF2 proteins are recruited to MARE sites in $\mathrm{HO}-1$ promoter activating its transcription in PC3 cell line.

\section{Discussion}

Prostate cancer continues to be a worldwide problem in the area of health, being the second cause of death by cancer in men (1). Many of the factors that take part in the development of prostate cancer, such as age, genetic predisposition, environmental factors, diet, and exposure to infectious agents or androgens, promote imbalance in the redox state of the cell (27), leading to tissue remodeling and proliferation. Chronic and acute inflammation generates ROS that damage cellular structures.

HO-1 plays a crucial role in the maintenance of cellular homeostasis and in the control of inflammation and oxidative stress. Based on this, we investigated the mechanisms of transcriptional regulation of HO-1, which still remain unknown.
Because of the essential role of BRCA1 in the maintenance of genomic integrity, we focused our work in the potential transcriptional regulation of $H O-1$ by BRCA1. We found that BRCA1 induces HO-1 expression in prostate cancer cell lines and modulates its function through transcriptional regulation of its target genes: UPA, MMP9, and Cyclin D1. Further, we demonstrate for the first time that BRCA1 associates with $\mathrm{HO}-1$ promoter at $4 \mathrm{~Kb}$ and $9 \mathrm{~Kb}$ upstream of the HO-1 TSS.

Herein, we propose that BRCA1 could fulfill its protective role counteracting oxidative stress through the transcriptional regulation of HO-1. Thus, the results exposed here support a new function for BRCA1 in tumor suppression of prostate cancer: the maintenance of the cellular homeostasis through the transcriptional activation of $\mathrm{HO}-1$.

Previously, Bae and colleagues (9) demonstrated that BRCA1 prevents DNA damage due to ionizing radiations and other sources by ROS detoxification. These evidences are in line with the results presented here where $\mathrm{HO}-1$ expression is modulated by 
diverse genotoxic and oxidating agents, as well as by the transcriptional regulator BRCA1 (9). HO-1 regulation by DNA-damage agents and/or oxidative stress inducers, herein stated, would affect the defense mechanism to maintain cellular homeostasis. The differential modulation obtained when cells are exposed either to DNA-damage inducers or to oxidative stress agents suggests the existence of a dual role for BRCA1. We speculate that BRCA1 is primarily recruited to $\mathrm{HO}-1$ promoter under oxidative injury, but under DNA damage, it becomes majorly recruited to other promoters different than the HO-1 gene.

In particular, we demonstrate that doxorubicin increases HO-1 levels in prostate cancer xenografts. Given that doxorubicin is used in chemotherapy for the treatment of several types of cancer, our results will contribute with additional evidence for $\mathrm{HO}-1$ 's potentiality as a therapeutic target. Future studies will determine the relevance of these findings in prostate cancer therapy or prognosis.

HO-1 role in the DNA-damage response has been previously reported (28). These authors showed that HO-1 modulates DNA repair through carbon monoxide. In this analysis, the authors described a new function for HO-1, modulating ATM protein expression. In addition, we previously reported that BRCA1 also regulates ATM expression (12). Altogether, the results presented here show for the first time a new BRCA1/HO-1/ATM axis regulation.

Regarding HO-1 cellular localization, previous works from our laboratory reported that HO-1 nuclear translocation is associated with prostate cancer (25). In this work, we demonstrated that BRCA1-overexpressing cells and/or cells exposed to doxorubicin do not display greater HO-1 translocation to the nucleus compared with cells with control levels of BRCA1 and cells that are not exposed to the chemotherapeutic agent. HO-1 is active at the cytoplasm $(26,29)$; therefore, the observed cytoplasmic HO-1 retention after BRCA1 overexpression and/or doxorubicin exposure could be associated with HO-1 function such as cellular homeostasis modulation.

To further our analysis on the HO-1/BRCA1/NRF2 axis, we used cBioPortal $(30,31)$ to mine genomic datasets of human prostate tumors. The cross-cancer alteration summary for BRCA1, HMOX1 (HO-1), and NFE2L2 (NRF2) displays no significant pattern for mutations, amplifications, deletions, or multiple alterations (Supplementary Fig. S1A). Of the nine datasets present, only one, the MSKCC-Prostate Oncogenome Project dataset (103 clinical human samples of prostate adenocarcinoma vs. normal gland), showed a statistically significant tendency toward co-occurrence of alterations in the gene pair BRCA1-NRF2 $(P=0.01)$. Regarding mRNA coexpression of HO-1/BRCA1/ NRF2, results display some discrepancies in Pearson correlation scores. While in the Broad/Cornell 2012 \& 2013 studies, BRCA1/
NRF2 and HO-1/NRF2 pairs show a high mRNA coexpression (Pearson $=0.56$ and 0.59 , respectively; Supplementary Fig. S1B), in the MSKCC study, HO-1/NRF2 mRNA coexpression is listed among the highest expression correlation pair but with a Pearson score of -0.44 (Supplementary Fig. S1C). mRNA expression zscores versus normal for the same pair also rank among the list of highest expression correlation with a similar Pearson correlation (-0.47; Supplementary Fig. S1D). Interestingly and in line with the work presented here, the HO-1/BRCA1 pair exhibits a high expression correlation when studying mRNA expression outliers (Pearson correlation 0.35; Supplementary Fig. S1E). In summary, we establish a new function for BRCA1 in the survival and fate of prostate tumor cells, orchestrating oxidative stress and mounting a transcriptional regulatory machinery commanding HO-1 transcriptional activation.

\section{Disclosure of Potential Conflicts of Interest \\ No potential conflicts of interest were disclosed.}

\section{Authors' Contributions}

Conception and design: E. Labanca, E. Vazquez, A. De Siervi

Development of methodology: E. Labanca, P. De Luca, C.P. Moiola, C. Massillo, A. De Siervi

Acquisition of data (provided animals, acquired and managed patients, provided facilities, etc.): E. Labanca, P. De Luca, G. Gueron, A. Paez, C.P. Moiola, J. Porretti, F. Zalazar, N. Navone, A. De Siervi

Analysis and interpretation of data (e.g., statistical analysis, biostatistics, computational analysis): E. Labanca, P. De Luca, G. Gueron, A. Paez, C.P. Moiola, J. Giudice, E. Vazquez, A. De Siervi

Writing, review, and/or revision of the manuscript: E. Labanca, P. De Luca, G. Gueron, A. Paez, C.P. Moiola, J. Giudice, E. Vazquez, A. De Siervi Administrative, technical, or material support (i.e., reporting or organizing data, constructing databases): E. Labanca, A. De Siervi Study supervision: P. De Luca, E. Vazquez, A. De Siervi

\section{Grant Support}

This research was supported by the Argentinean Agency of Science and Technology (ANPCyT PICT 2013-2152; ANPCyT PICT 2012-00374) and the NCI (2011) from Argentina. We thank the Fundacion Williams (Argentina) and Fundacion Rene Barón (Argentina) for their support. We are grateful to the Prostate Cancer Foundation (USA) for the Young Investigator Award 2013 given to Geraldine Gueron. J. Giudice is a Pew Latin American Fellow in the Biomedical Sciences supported by The Pew Charitable Trusts (\#2933). This work was completed for the undergraduate thesis of E. Labanca.

The costs of publication of this article were defrayed in part by the payment of page charges. This article must therefore be hereby marked advertisement in accordance with 18 U.S.C. Section 1734 solely to indicate this fact.

Received March 30, 2015; revised June 24, 2015; accepted July 22, 2015 published OnlineFirst July 30, 2015.

\section{References}

1. Jemal A, Bray F, Center MM, Ferlay J, Ward E, Forman D. Global cancer statistics. CA Cancer J Clin 2011;61:69-90.

2. Bickers B, Aukim-Hastie C. New molecular biomarkers for the prognosis and management of prostate cancer-the post PSA era. Anticancer Res 2009;29:3289-98

3. De Marzo AM, Platz EA, Sutcliffe S, Xu J, Gronberg H, Drake CG, et al. Inflammation in prostate carcinogenesis. Nat Rev Cancer 2007; 256-69.

4. Debatin KM, Poncet D, Kroemer G. Chemotherapy: targeting the mitochondrial cell death pathway. Oncogene 2002;21: 8786-803.

5. Pelicano H, Carney D, Huang P. ROS stress in cancer cells and therapeutic implications. Drug Resist Updat 2004;7:97-110.

6. Martindale JL, Holbrook NJ. Cellular response to oxidative stress: signaling for suicide and survival. J Cell Physiol 2002;192:1-15.

7. D'Autreaux B, Toledano MB. ROS as signalling molecules: mechanisms that generate specificity in ROS homeostasis. Nat Rev Mol Cell Biol 2007;8: 813-24.

8. Sherr CJ. Principles of tumor suppression. Cell 2004;116:235-46.

9. Bae I, Fan S, Meng Q, Rih JK, Kim HJ, Kang HJ, et al. BRCA1 induces antioxidant gene expression and resistance to oxidative stress. Cancer Res 2004;64:7893-909. 
Labanca et al.

10. De Siervi A, De Luca P, Byun JS, Di LJ, Fufa T, Haggerty CM, et al. Transcriptional autoregulation by BRCA1. Cancer Res 2010;70: $532-42$.

11. De Luca P, Vazquez ES, Moiola CP, Zalazar F, Cotignola J, Gueron G, et al. BRCA1 loss induces GADD153-mediated doxorubicin resistance in prostate cancer. Mol Cancer Res 2011;9:1078-90.

12. Moiola C, De Luca P, Cotignola J, Gardner K, Vazquez E, De Siervi A. Dynamic coregulatory complex containing BRCA1, E2F1 and CtIP controls ATM transcription. Cell Physiol Biochem 2012;30:596-608.

13. Gueron G, De Siervi A, Vazquez E. Key questions in metastasis: new insights in molecular pathways and therapeutic implications. Curr Pharm Biotechnol 2011;12:1867-80

14. Ferrando M, Gueron G, Elguero B, Giudice J, Salles A, Leskow FC, et al. Heme oxygenase 1 (HO-1) challenges the angiogenic switch in prostate cancer. Angiogenesis 2011;14:467-79.

15. Poss KD, Tonegawa S. Reduced stress defense in heme oxygenase 1deficient cells. Proc Natl Acad Sci U S A 1997;94:10925-30.

16. Yachie A, Niida Y, Wada T, Igarashi N, Kaneda H, Toma T, et al. Oxidative stress causes enhanced endothelial cell injury in human heme oxygenase-1 deficiency. J Clin Invest 1999;103:129-35.

17. Gueron G, De Siervi A, Ferrando M, Salierno M, De Luca P, Elguero B, et al. Critical role of endogenous heme oxygenase 1 as a tuner of the invasive potential of prostate cancer cells. Mol Cancer Res 2009;7:1745-55.

18. Martin D, Rojo AI, Salinas M, Diaz R, Gallardo G, Alam J, et al. Regulation of heme oxygenase-1 expression through the phosphatidylinositol 3-kinase/Akt pathway and the Nrf2 transcription factor in response to the antioxidant phytochemical carnosol. J Biol Chem 2004;279:8919-29.

19. De Luca P, Moiola CP, Zalazar F, Gardner K, Vazquez ES, De Siervi A. BRCA1 and p53 regulate critical prostate cancer pathways. Prostate Cancer Prostatic Dis 2013;16:233-8.

20. Aprelikova ON, Fang BS, Meissner EG, Cotter S, Campbell M, Kuthiala A, et al. BRCA1-associated growth arrest is RB-dependent. Proc Natl Acad Sci U S A 1999;96:11866-71.
21. Elguero B, Gueron G, Giudice J, Toscani MA, De Luca P, Zalazar F, et al Unveiling the association of STAT3 and HO-1 in prostate cancer: role beyond heme degradation. Neoplasia 2012;14:1043-56.

22. Giudice J, Jares-Erijman EA, Leskow FC. Endocytosis and intracellular dissociation rates of human insulin-insulin receptor complexes by quantum dots in living cells. Bioconjug Chem 2013;24:431-42.

23. Giudice J, Barcos LS, Guaimas FF, Penas-Steinhardt A, Giordano L, JaresErijman EA, et al. Insulin and insulin like growth factor II endocytosis and signaling via insulin receptor B. Cell Commun Signal 2013;11:18

24. Lidke DS, Nagy P, Heintzmann R, Arndt-Jovin DJ, Post JN, Grecco HE, et al. Quantum dot ligands provide new insights into erbB/HER receptor-mediated signal transduction. Nat Biotechnol 2004;22:198-203.

25. Sacca P, Meiss R, Casas G, Mazza O, Calvo JC, Navone N, et al. Nuclear translocation of haeme oxygenase-1 is associated to prostate cancer. $\mathrm{Br} \mathrm{J}$ Cancer 2007;97:1683-9.

26. Wegiel B, Gallo D, Csizmadia E, Harris C, Belcher J, Vercellotti GM, et al. Carbon monoxide expedites metabolic exhaustion to inhibit tumor growth. Cancer Res 2013;73:7009-21.

27. Klein EA, Casey G, Silverman R. Genetic susceptibility and oxidative stress in prostate cancer: integrated model with implications for prevention. Urology 2006;68:1145-51.

28. Otterbein LE, Hedblom A, Harris C, Csizmadia E, Gallo D, Wegiel B. Heme oxygenase- 1 and carbon monoxide modulate DNA repair through ataxiatelangiectasia mutated (ATM) protein. Proc Natl Acad Sci U S A 2011; 108:14491-6.

29. Lin QS, Weis S, Yang G, Zhuang T, Abate A, Dennery PA. Catalytic inactive heme oxygenase-1 protein regulates its own expression in oxidative stress. Free Radic Biol Med 2008;44:847-55.

30. Gao J, Aksoy BA, Dogrusoz U, Dresdner G, Gross B, Sumer SO, et al. Integrative analysis of complex cancer genomics and clinical profiles using the cBioPortal. Sci Signal 2013;6:pl1.

31. Cerami E, Gao J, Dogrusoz U, Gross BE, Sumer SO, Aksoy BA, et al. The cBio cancer genomics portal: an open platform for exploring multidimensional cancer genomics data. Cancer Discov 2012;2:401-4. 


\section{Molecular Cancer Research}

\section{Association of HO-1 and BRCA1 Is Critical for the Maintenance of Cellular Homeostasis in Prostate Cancer}

Estefanía Labanca, Paola De Luca, Geraldine Gueron, et al.

Mol Cancer Res 2015;13:1455-1464. Published OnlineFirst July 30, 2015.

Updated version Access the most recent version of this article at:

doi:10.1158/1541-7786.MCR-15-0150-T

Cited articles This article cites 31 articles, 12 of which you can access for free at:

http://mcr.aacrjournals.org/content/13/11/1455.full.html\#ref-list-1

E-mail alerts Sign up to receive free email-alerts related to this article or journal.

Reprints and To order reprints of this article or to subscribe to the journal, contact the AACR Publications Department at Subscriptions pubs@aacr.org.

Permissions To request permission to re-use all or part of this article, contact the AACR Publications Department at permissions@aacr.org. 